\title{
Drug Cost Avoidance Analysis of Cancer Clinical Trials in Spain: A Study on Cost Contributors and Their Impact
}

\section{Sánchez-Martínez Domingo}

Hospital Clínico Universitario Virgen de la Arrixaca. IMIB-Arrixaca. Medical Oncology Service Salas-Lucia Federico ( $\square$ fsalaslucia@uchicago.edu )

The University of Chicago

Jiang Hanzi

West Side United

\section{Ruiz-Carreño Paula}

Hospital Clínico Universitario Virgen de la Arrixaca. IMIB-Arrixaca. Medical Oncology Service Alonso Romero José Luis

Hospital Clínico Universitario Virgen de la Arrixaca. IMIB-Arrixaca. Medical Oncology Service

\section{Research Article}

Keywords: Hospital management, Linear regression, Healthcare costs, Sustainability, Clinical Trials, Oncology

Posted Date: January 24th, 2022

DOI: https://doi.org/10.21203/rs.3.rs-1287447/v1

License: (c) (i) This work is licensed under a Creative Commons Attribution 4.0 International License. Read Full License 


\title{
Drug Cost Avoidance Analysis of Cancer Clinical Trials in Spain: A Study on Cost Contributors and Their Impact
}

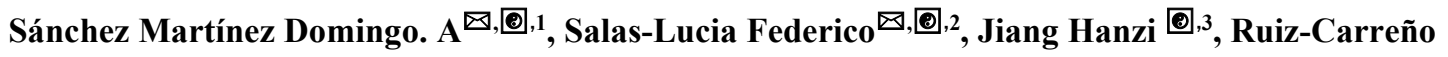 \\ Paula $^{1}$ y Alonso Romero José Luis ${ }^{1}$ \\ 1 Hospital Clínico Universitario Virgen de la Arrixaca. IMIB-Arrixaca. Medical Oncology Service; \\ Murcia, Spain. \\ 2 Department of Medicine, The University of Chicago, Chicago, IL, USA. \\ 3 West Side United, Chicago, IL, USA \\ Correspondence authors: domingoa.sanchez@carm.es, fsalaslucia@uchicago.edu. \\ (2) Authors contributed equally
}

\begin{abstract}
Objective: Analyze the cost contributors and their impact on the drug cost avoidance (DCA) resulting from cancer clinical trials over the period of 2015-2020 in a tertiary-level hospital in Spain (HCUVA). Methods: We performed a cross-sectional, observational, retrospective study of a total of 53 clinical trials with 363 patients enrolled. We calculated the DCA from the price of the best standard of care (i.e.: drugs that the institution would otherwise fund). A linear regression model was used to determine cost contributors and estimate their impact. Results: The total DCA was $\sim 5.1$ million euros ( 31 clinical trials; 177 enrollees), representing $30 \%$ and $0,05 \%$ approximately of the annual pharmaceutical expenditures at the HCUVA and for the Spanish Health System, respectively. Cancer type analysis showed that lung cancer had the highest average DCA by trial, indicating that treatments in these trials were the most expensive. Linear regression analysis showed that the number of patients in a trial did not significantly affect that trial's DCA. Instead, cancer type, phase trials, and intention of treatment were significant cost contributors to DCA. Compared to digestive cancer trials, breast and lung trials were significantly more expensive, $(\mathrm{p}<0.05$ and $\mathrm{p}<0.1$, respectively). Phase III trials were more expensive than Phase II $(p<0.05)$ and adjuvant trials were less expensive than palliative $(p<0.001)$. Conclusion: We identified key cancer clinical trial characteristics that significantly impacted the estimated DCA. Our work provides the groundwork to explore DCA contributors with potential to enhance public relations material and serve as a negotiating tool for budgeting, thus playing an important role to inform decisions about resource allocation.
\end{abstract}

Keywords: Hospital management, Linear regression, Healthcare costs, Sustainability, Clinical Trials, Oncology.

\section{Introduction}

Cancer has one of the most significant impacts on health budgets ${ }^{1,2}$, and estimations predict that the number of new cases will increase over the next two decades to 29.5 million per year by $2040^{3}$. Worryingly, the lack of situation analysis and budgeting concerning this pathology has been identified as one of the main obstacles threatening the sustainability of health systems ${ }^{4}$. Recent studies propose clinical trials as an alternative new element to introduce sustainability into the 
Health System ${ }^{5}$, aiming to prevent economic failure. Clinical trials investigate new diagnosing, treating, and managing cancer to improve the standard of care treatment ${ }^{6}$. For patients, the benefits of clinical trials include gaining access to experimental treatments when no other options exist and to new therapies not yet available ${ }^{7}$, while contributing to the advancement of medical research. However, the economic benefits of running oncologic clinical trials for hospital administrators are often not considered. One potential financial benefit of clinical trials is drug cost avoidance $(\mathrm{DCA})^{8,9}$, resulting when trial subjects receive industry-sponsored treatment drugs that the institution would otherwise fund ${ }^{10}$. Previous studies have quantified DCA derived from cancer clinical trials in different settings ${ }^{11,12}$, proven their efficacy to alleviate the economic pressure in the Health system. However, there are no studies on cost contributors and their impact on DCA to the best of our knowledge. The purpose of this study was to analyze the cost contributors and their impact on the DCA resulting from cancer clinical trials throughout 20152020 in a tertiary-level hospital in Spain (HCUVA). We calculated the DCA from the price of the best standard of care. The total DCA was $\sim 5.1$ million euros, representing 30\% and approximately the $0,05 \%$ of the annual pharmaceutical expenditures at the HCUVA and for the Spanish Health System, respectively. Cancer type, phase trials, and intention of treatment were significant cost contributors to DCA. Breast and lung trials were significantly more expensive than digestive cancer trials $(\mathrm{p}<0.05$ and $\mathrm{p}<0.1$, respectively). Phase III trials were more expensive than Phase II $(\mathrm{p}<0.05)$ and adjuvant trials were less expensive than palliative $(\mathrm{p}<0.001)$. Our work provides the groundwork to explore DCA contributors with the potential to enhance public relations material and serve as a negotiating tool for budgeting, thus playing an essential role in informing resource allocation decisions.

\section{Methods}

\subsection{Identifying eligible trials}

We reviewed clinical trials performed from 2015 to 2020 at the oncology department of HCUVA. Trials were excluded when (i) no patients were enrolled, (ii) screening failed, (iii) DCA could not be calculated due to insufficient drug information, and (iv) were observational phase 4 trials in which the experimental treatment was not industry-sponsored.

\subsection{Calculating DCA}

Pharmacy and patient records for eligible trials were reviewed to determine each subject's treatment duration and quantity of the drug. Because the cost of the experimental drug in the clinical trials was unavailable, we calculated the best standard of care DCA using a reference price list. When the standard of care drug dosage depends on the body surface area, we used standardized parameters for men and women $(1.96 \mathrm{~kg} / \mathrm{m} 2$ and $1.68 \mathrm{~kg} / \mathrm{m} 2$, respectively). Equation [1] shows how we calculated the DCA. 


\subsection{Linear regression analysis}

We constructed a regression model using four control variables: number of patients, cancer type, trial phases, and intention of treatment. Equation [2] shows our regression model. We set our baseline constant as digestive cancer, palliative treatment, and Phase II trials ( $\beta_{0}$; namely the intercept) to establish a comparison for the indicator variables. The dependent variable was the DCA from each trial. The variable number of patient (npatients) is numeric, and the other three variables are composed of characteristic values. We then created indicator variables to translate characteristic values into numeric (e.g., indicator column for breast cancer will indicate breast cancer as 1 , other cancer types as 0 ). Using the ' $1 \mathrm{~m}$ ' function of the $\mathrm{R}$ statistic software, the coefficient estimates $(\beta)$ of each variable represents the magnitude of its impact on trial DCA while holding other factors constant.

$$
D C A=\beta_{0}+\beta_{1} \text { npatients }+\beta_{2} \text { CancerType }+\beta_{3} \text { Intention }+\beta_{4} \text { Phase }+\varepsilon_{i}
$$

\section{Results}

\subsection{Study sample}

We reviewed 53 industry-sponsored clinical trials with a total of 363 patients enrolled, from 2015 to 2020, at the oncology department of HUCVA. We excluded three trials that had no patient enrolled, 13 trials ( 65 patients) were in phase IV, for six trials (21 patients), we could not calculate the DCA and 100 patients failed the screening. The final sample studied included 31 trials, enrolling 177 patients (Figure 1). The number of patients per study ranged from 1 to 17. Trials were distributed among seven tumor groups; the most common was breast cancer (13 trials), followed by digestive (6), gynecology (5), lung (4), prostate (1), melanoma (1), and sarcoma (1). The final sample had phase II and III trials ( 7 and 24, respectively) with palliative (25), adjuvant (4) and neoadjuvant (2) intentions.

\subsection{Drug cost avoidance}

The DCA in all trials was calculated by the price of the standard treatment, including a total of 35 drugs. The estimated DCA during the study period was $\sim 5.1$ million euros (Table 1). The median estimated DCA per year was $962,272 €$ and $36,294 €$ per trial. DCA in this study represents approximately $30 \%$ and $0,05 \%$ of the annual pharmaceutical expenditures at the Medical Oncology Department at HCUVA and for the Spanish Health System (15), respectively (The hospital pharmacy service provided us with the pharmaceutical budget for the HCUVA). We found that DCA did not increase proportionally to the number of trials or number of patients (Table 2). For instance, in 2016, the DCA was 1,6 million euros from 4 trials and 23 patients, while in 2019, it was 0.9 million euros from 9 trials and 59 patients. Only $6.5 \%$ of DCA came 
from Phase II and 93.5\% from phase III. Regarding the intention of clinical trials, palliative trials represented $89.2 \%$ of the DCA, followed by neoadjuvant and adjuvant $(9.3 \%$ and $1.5 \%$, respectively). Next, we conducted a cancer type analysis of the DCA and found that breast cancer had the highest DCA (Table 3). However, lung cancer had the highest average DCA by trial, and it was twice as much the breast cancer's, indicating that, on average, lung cancer treatments were the most expensive (Table 3). Digestive cancer had the least expensive treatments on average.

\subsection{Cost contributor's analysis}

Next, we wished to investigate the impact magnitude of each cost contributor on the DCA. Our regression model showed that the number of patients in a trial did not significantly affect that trial's DCA (Table 4). However, we found a significant impact on cancer type, phase trials, and intention of treatment (Table 4). Specifically, compared to digestive cancer trials, breast and lung cancer trials were 587,640 $€(\mathrm{p}<0.05)$ and 381,678 $€(\mathrm{p}<0.1)$ more expensive, respectively. Similarly, Phase III trials were 539,305 $€(p<0.05)$ more expensive than Phase II, and regarding the intention of the trials, adjuvant trials were $751,058 €(\mathrm{p}<0.001)$ less expensive than palliative (Table 4).

\section{Discussion}

This paper estimated the DCA from industry-sponsored oncological clinical trials that took place in HCUVA between 2015 and 2020. To estimate the DCA, we used the price of the best standard of care, resulting in $~ 5.1$ million euros (Table 1). Other DCA estimations performed in a single institution have shown similar saving percentages for the hospital or institution budget ${ }^{11,12}$. Thus, supporting the idea that the DCA from oncological clinical trials provides economic savings, and may help to sustain the national health system. A limitation of our and previous studies is that other non-pharmaceutical costs were not considered, implying the savings derived from oncological clinical trials could be underestimated. Additionally, our study contained a unique sample of trials that had a diverse number of enrollees, cancer types, intentions of the treatments and phase, making our DCA estimations difficult to be directly compared with other estimated DCA. For instance, previous studies have estimated DCA by variables such as the number of patients, the length, or phases of the trials ${ }^{11}$, the pharmacological categories ${ }^{13,14}$ or pathology type ${ }^{14}$.

Our initial data exploration showed that variations in the number of clinical trials and patients enrolled per year did not represent a proportional variation in the annual DCA (Table 2), incentivizing us to further investigate other potential DCA contributing factors. Our cancer type analysis of the DCA showed that lung cancer trials had the highest average DCA per trial compared with other cancer types, suggesting lung cancer treatments could be the most expensive 
(Table 3). This agrees with the observation that the DCA per patient varies drastically among tumor groups ${ }^{15}$. We suspected that other trial characteristics, such as treatment intention and phases, could also have significant impacts on DCA. Therefore, to further investigate and isolate each individual characteristic's impact on DCA, we constructed a linear regression model as illustrated in equation [2]. The linear regression results (Table 4) shows that the number of patients enrolled in a trial had a trivial contribution to DCA, which agrees with our previous observation (Table 2). Thus, suggesting other contributors could carry more weight on influencing DCA. Indeed, our linear regression analysis confirmed that certain cancer types, trial phases, and treatment intention had a significant impact on DCA (Table 4). Specifically, we found that palliative trials tend to be $751,058 €(\mathrm{p}<0.001)$ more expensive than adjuvant trials while holding other variables constant. Similarly, breast and lung cancer trials were 587,640€ (p $<0.05)$ and $381,678 €(\mathrm{p}<0.1)$ more expensive than digestive cancer trials, respectively. These findings could be explained by the varying dosage requirements for differing trials, the length of time patients remain on trial drugs due to progression of disease, and the cost of the treatments. For example, in our study, each treatment cycle's price between trials ranged from $43 €$ to 8640 $€$ (Table 1). These price differences reflect that innovative therapies, such as cyclin inhibitors and immunotherapy, are significantly more costly than other standard chemotherapies ${ }^{11,16}$. The increasing number of new options for prevention, diagnosis, and treatment, makes studies of the DCA of the new therapies increasingly important to inform decisions about resource allocation. Our study might be useful for hospital management by providing a projection on future DCA derived from clinical trials based on their characteristics.

\section{Conclusion}

We identified key cancer clinical trial characteristics that significantly impacted the estimated DCA. Our work provides the groundwork to explore DCA contributors with the potential to enhance public relations material and serve as a negotiating tool for budgeting, thus playing an essential role in informing resource allocation decisions.

Acknowledgements: Hanzi Jiang is a recipient of the Applied Data Fellowship from the Harris School of Public Policy, The University of Chicago.

Funding: This research did not receive any specific grant from funding agencies in the public, commercial, or not-for-profit sectors.

Disclosure: The authors have declared no conflicts of interest.

Author contributions: PR-C, JLAR and DASM studied the databases and identified eligible trials. DASM and JLAR estimated the DCA and analyzed the trial's distribution. HJ performed 
the linear regression study and prepared the figures and tables. HJ wrote the first draft of the paper. DASM and FS-L supervised the study. FS-L finalized the manuscript for review by all authors.

\section{Bibliography}

1. Gourd E. Impact of the UK Budget on cancer care and research. Lancet Oncol. Dec 2021;22(12):1659. doi:10.1016/S1470-2045(21)00645-8

2. Luengo-Fernandez R, Leal J, Gray A, Sullivan R. Economic burden of cancer across the European Union: a population-based cost analysis. Lancet Oncol. Nov 2013;14(12):1165-74. doi:10.1016/S1470-2045(13)70442-X

3. Ferlay J, Colombet M, Soerjomataram I, et al. Estimating the global cancer incidence and mortality in 2018: GLOBOCAN sources and methods. Int J Cancer. 0415 2019;144(8):1941-1953. doi:10.1002/ijc.31937

4. Fitzmaurice C, Abate D, Abbasi N, et al. Global, Regional, and National Cancer Incidence, Mortality, Years of Life Lost, Years Lived With Disability, and DisabilityAdjusted Life-Years for 29 Cancer Groups, 1990 to 2017: A Systematic Analysis for the Global Burden of Disease Study. JAMA Oncol. 1201 2019;5(12):1749-1768. doi:10.1001/jamaoncol.2019.2996

5. Zinzani PL, Dreyling M, Gradishar W, et al. Are Biosimilars the Future of Oncology and Haematology? Drugs. Oct 2019;79(15):1609-1624. doi:10.1007/s40265019-01193-y

6. Krzyzanowska MK, Kaplan R, Sullivan R. How may clinical research improve healthcare outcomes? Ann Oncol. Nov 2011;22 Suppl 7:vii10-vii15. doi:10.1093/annonc/mdr420

7. Buffery D. The 2015 Oncology Drug Pipeline: Innovation Drives the Race to Cure Cancer. Am Health Drug Benefits. Jun 2015;8(4):216-22.

8. Herledan C, Ranchon F, Schwiertz V, et al. Drug cost savings in phase III hematological oncology clinical trials in a university hospital. Hematol Oncol. Oct 2020;38(4):576-583. doi:10.1002/hon.2753

9. Jones B, Syme R, Eliasziw M, Eigl BJ. Incremental costs of prostate cancer trials: Are clinical trials really a burden on a public payer system? Can Urol Assoc J. 2013 MarApr 2013;7(3-4):E231-6. doi:10.5489/cuaj.11302

10. LaFleur J, Tyler LS, Sharma RR. Economic benefits of investigational drug services at an academic institution. Am J Health Syst Pharm. Jan 01 2004;61(1):27-32. doi:10.1093/ajhp/61.1.27

11. Mañes-Sevilla M, Romero-Jiménez R, Herranz-Alonso A, et al. Drug cost avoidance in clinical trials of breast cancer. J Oncol Pharm Pract. Jul 2019;25(5):10991104. doi:10.1177/1078155218775193

12. D'Ambrosio F, De Feo G, Botti G, et al. Clinical trials and drug cost savings for Italian health service. BMC Health Serv Res. Nov 26 2020;20(1):1089. doi:10.1186/s12913-020-05928-6

13. Shen LJ, Chou H, Huang CF, Chou GM, Chan WK, Wu FL. Economic benefits of sponsored clinical trials on pharmaceutical expenditures at a medical center in Taiwan. Contemp Clin Trials. Jul 2011;32(4):485-91. doi:10.1016/j.cct.2011.04.003

14. Tang PA, Hay AE, O'Callaghan CJ, et al. Estimation of drug cost avoidance and pathology cost avoidance through participation in NCIC Clinical Trials Group phase III clinical trials in Canada. Curr Oncol. Feb 2016;23(Suppl 1):S7-S13. doi: $10.3747 /$ co.23.2861 
15. Bredin C, Eliasziw M, Syme R. Drug cost avoidance resulting from cancer clinical trials. Contemp Clin Trials. Nov 2010;31(6):524-9. doi:10.1016/j.cct.2010.09.004

16. Verma V, Sprave T, Haque W, et al. A systematic review of the cost and costeffectiveness studies of immune checkpoint inhibitors. J Immunother Cancer. 1123 2018;6(1):128. doi:10.1186/s40425-018-0442-7 
Figures

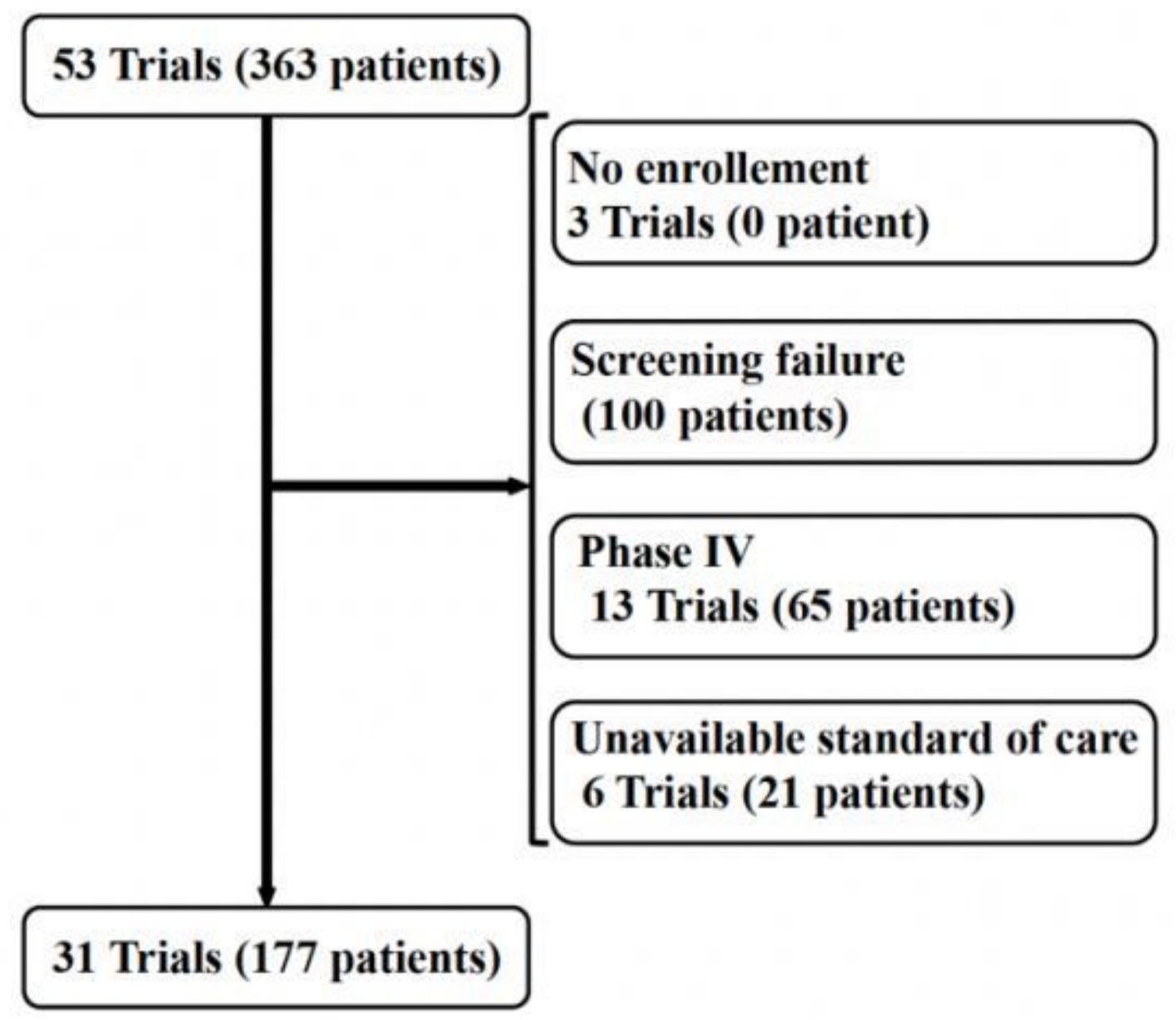

Figure 1

Flow chart of the process to identify eligible trials.

\section{Supplementary Files}

This is a list of supplementary files associated with this preprint. Click to download.

- Table1.pdf

- Table2.pdf

- Table3.pdf

- Table4.pdf 